\title{
ANÁLISE DE TEMPESTADES GEOMAGNÉTICAS SUPER INTENSAS E DE ESTRUTURAS DO MEIO INTERPLANETÁRIO RELACIONADAS, ATRAVÉS DA OBSERVAÇÃO DE RAIOS CÓSMICOS DE SUPERFÍCIE DE ALTA ENERGIA
}

\author{
Jairo Francisco Savian ${ }^{1}$, Marlos Rockenbach da Silva², Alisson Dal Lago ${ }^{3}$, Kazuoki Munakata ${ }^{4}$, \\ Walter Demetrio Gonzalez ${ }^{5}$ e Nelson J. Schuch ${ }^{6}$ \\ Recebido em 22 março, 2005 / Aceito em 22 agosto, 2005 \\ Received on March 22, 2005 / Accepted on August 22, 2005
}

\begin{abstract}
It is believed that the physical mechanism responsible for the transference of energy from the solar wind to the Earth magnetosphere is the reconnection between the interplanetary magnetic field and the terrestrial magnetic field (Tsurutani \& Gonzalez, 1997). The necessary criterion for a Intense Geomagnetic Storms to occur, Dst $<-100 \mathrm{nT}$, is the existence of a dawn-dusk interplanetary electric field larger than $5 \mathrm{mV} / \mathrm{m}$, for a period larger than 3 hours. Cosmic rays have been studied as a natural phenomenon that can tell us much about both Earth's environment in space and distant astrophysical processes (Jokipii, 2000). A solar disturbance propagating away from the Sun affects the pre-existing population of galactic cosmic rays in a number of ways. The most famous one is known as the "Forbush decrease", which is a supression of ground cosmic-ray counts observed during Geomagnetic Disturbances. The objective of this work is to study the response of the Southern Space Observatory - SSO ground Muon Telescope observations, installed in São Martinho da Serra, RS, Brazil, to 3 super intense geomagnetic storms, combining observation provided by L1 satellites and ground detectors.
\end{abstract}

Keywords: interplanetary medium, cosmic rays, Muon Telescope, Forbush decreases, space weather, magnetic storms.

RESUMO. Acredita-se que o mecanismo físico responsável pela transferência de energia do vento solar para a magnetosfera terrestre seja a reconexão entre 0 campo magnético interplanetário e o campo magnético terrestre (Tsurutani \& Gonzalez, 1997). 0 critério necessário para que ocorra uma Tempestade Geomagnética Intensa, Dst <-100nT, é o de haver um campo elétrico interplanetário, na direção do anoitecer, maior que $5 \mathrm{mV} / \mathrm{m}$, por um período maior que 3 horas. Raios cósmicos são fenômenos naturais que podem nos ensinar muito tanto sobre o espaço vizinho à Terra, quanto sobre processos astrofísicos distantes (Jokipii, 2000). As estruturas solares que se propagam no meio interplanetário afetam a população pré-existente de raios cósmicos galácticos de várias maneiras. A mais conhecida é 0 chamado "decréscimo de Forbush", que é uma diminuição das contagens de raios cósmicos observados na superfície durante distúrbios Geomagnéticos. 0 objetivo deste trabalho é estudar a resposta das observações do Telescópio Cintilador Multidirecional de Muons do Observatório Espacial do Sul - OES/CRSPE/INPE-MCT, instalado em São Martinho da Serra, RS, Brasil, a 3 Tempestades Geomagnéticas Super Intensas, combinando observações feitas por satélites localizados no ponto Lagrangeano L1, e detectores de superfície.

\footnotetext{
Palavras-chave: meio interplanetário, raios cósmicos, Telescópio Cintilador de Muons, decréscimo de "Forbush", Clima Espacial, Tempestades Geomagnéticas.

${ }^{1}$ Centro Regional Sul de Pesquisas Espaciais - CRSPE/INPE-MCT, Faixa de Camobi, Km 9, Campus Universitário, 97105-900 Santa Maria, RS. Tel: 55-3220-8021 -E-mail: savian@lacesm.ufsm.br

2 Instituto Nacional de Pesquisas Espaciais - INPE-MCT, Av. dos Astronautas, 1.758 - Jd. Granja, 12227-010 São José dos Campos, SP. Tel: 12-3945-6808 -E-mail: marlos@dge.inpe.br

3 Instituto Nacional de Pesquisas Espaciais - INPE-MCT, Av. dos Astronautas, 1.758 - Jd. Granja, 12227-010 São José dos Campos, SP. Tel: 12-3945-6979 -E-mail: dallago@dge.inpe.br

${ }^{4}$ Physics Department, Shinshu University, Matsumoto, Japan

${ }^{5}$ Instituto Nacional de Pesquisas Espaciais - INPE-MCT, Av. dos Astronautas, 1.758 - Jd. Granja, 12227-010 São José dos Campos, SP. Tel: 12-3945-6979 -E-mail: gonzalez@dge.inpe.br

${ }^{6}$ Centro Regional Sul de Pesquisas Espaciais - CRSPE/INPE-MCT, Faixa de Camobi, Km 9, Campus Universitário, 97105-900 Santa Maria, RS. Tel: 55-3220-8021 -E-mail: njschuch@lacesm.ufsm.br
} 


\section{INTRODUÇÃO}

A cada 11 anos 0 Sol passa por um período de mínima e outro de máxima atividade magnética, sendo este último denominado Máximo Solar, apresentando um maior número de instabilidades, as quais liberam grandes quantidades de matérias na forma de Ejeções Coronal de Massa (do inglês: Coronal Mass Ejection - CME). Estas manifestações de intensa atividade atingem o meio interplanetário e os planetas causando as chamadas Tempestades Geomagnéticas proporcionando um transporte efetivo de energia para a magnetosfera, culminando com um aumento na densidade de partículas que circundam a Terra. Estes distúrbios solares afetam a população de raios cósmicos galácticos préexistentes de várias maneiras. A mais conhecida é 0 "decréscimo de Forbush", uma região de supressão da densidade de raios cósmicos localizadas atrás de um choque devido a uma Ejeção Coronal de Massa. Os efeitos mais conhecidos das Tempestades Geomagnéticas são: intensificação de correntes elétricas na Magnetosfera e na superfície terrestre, ocorrência de auroras nas regiões polares, aceleração de partículas carregadas, e diversos prejuízos em satélites causando danos no Sistema de Posicionamento Global (GPS) em telecomunicações e até mesmo em astronautas que se encontram em naves espaciais, devido a alta radiação emitida. Atualmente, a previsão da chegada na Terra de estruturas solares que podem causar Tempestades Geomagnéticas pode ser feita com antecedência de: (1) até 3 dias, utilizando observações do Sol feitas por satélites (com erros da ordem de \pm 1 dia); (2) até uma hora, utilizando observações de satélites no Ponto Lagrangeano L1 (com erro de alguns minutos). Num futuro próximo, pretende-se fazer previsões da ocorrência de distúrbios geomagnéticos com antecedência de oito a doze horas utilizando a Rede Internacional de Detectores de Muons, da qual fazem parte 3 Telescópios Cintiladores Multidirecionais de Muons (TCM): Nagoya (Japão), Hobart (Austrália) e São Martinho da Serra (Brasil). Como preparação para esta etapa futura, este trabalho apresenta um estudo preliminar do tipo de resposta apresentada pelos raios cósmicos de alta energia observados pelo TCM do Observatório Espacial do Sul - OES/CRSPE/INPE-MCT, em São Martinho da Serra, RS, Brasil, em relação a 3 Super Tempestades Geomagnéticas observadas em Outubro e Novembro de 2003.

\section{Tempestades Geomagnéticas}

Tempestades Geomagnéticas são eventos nos quais uma grande quantidade de energia é transferida do vento solar para dentro da Magnetosfera Terrestre, que é a região onde o Campo Geo- magnético controla os processos físicos, havendo precipitação de partículas energéticas do vento solar nas regiões aurorais, intensificando as correntes elétricas tanto na Magnetosfera quanto na superfície da Terra, tendo recebido esta denominação desde a metade do século XIX (Gonzalez et al., 1994). A característica principal de uma Tempestade Geomagnética é 0 decréscimo acentuado da componente horizontal, H, do Campo Geomagnético possuindo uma posterior fase de recuperação (Kamide et al., 1998). Este decréscimo é devido a um aumento da população de partículas aprisionadas na Magnetosfera. Derivas devidas ao gradiente do campo magnético e a sua curvatura produzem a chamada "Corrente de Anel".

A perturbação do campo geomagnético gerada por essa corrente está portanto, correlacionada às variações do campo magnético (CMI) e do plasma do meio interplanetário (vento solar), que controlam 0 suprimento de energia para 0 interior da Magnetosfera (Akasofu \& Chapman, 1972; Burton et al., 1975; Nishida, 1978; Lyons \& Williams, 1980).

Acredita-se que o mecanismo físico responsável pela transferência de energia do vento solar para a Magnetosfera seja a reconexão entre 0 campo magnético interplanetário e 0 campo magnético terrestre (Tsurutani \& Gonzalez, 1997). Para que aconteça este fenômeno é necessário que a componente do CMI esteja na direção antiparalela a do campo magnético terrestre (Figura 1). 0 critério necessário para que ocorra uma Tempestade Geomagnética Intensa, Dst <-100nT, é 0 de haver um campo elétrico interplanetário, $\vec{E}=\vec{V} \times \vec{B}_{s}$, na direção do anoitecer, maior que $5 \mathrm{mV} / \mathrm{m}$, por um período maior que 3 horas. Esse critério foi empiricamente definido por Gonzalez \& Tsurutani (1987), com dados de plasma e campo magnético obtidos pelo satélite ISEE-3, e implica, por exemplo, em um campo magnético Bs menor que $-12,5 \mathrm{nT}$ para uma velocidade do vento solar de aproximadamente $400 \mathrm{~km} / \mathrm{s}$.

\section{MATERIAL E MÉTODOS}

A frequêencia de ocorrência de Tempestades Geomagnéticas Super Intensas (Dst <-200nT) não é muito grande, variando de anos sem nenhum evento, a anos com cerca de 3 eventos (Tsurutani et al., 1992; Dal Lago et al., 2004). 0 presente trabalho tem por objetivo apresentar uma análise de 3 eventos solar-interplanetáriogeomagnéticos super intensos que ocorreram no ano de 2003. Para a análise dos dados, primeiramente utilizou-se observações de índice Dst disponíveis no sítio eletrônico da Universidade de Kyoto (Japão), que mede a intensidade da corrente de anel magnetosférica, para identificar as Tempestades Geomagnéticas mais 


\section{ACOPLAMENTO SOL-MEIO INTERPLANETÁRIO-MAGNETOSFERA}

\section{Magnetosfera Terrestre}

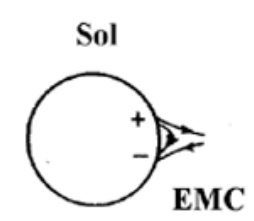

EMC: Ejeção de massa coronal
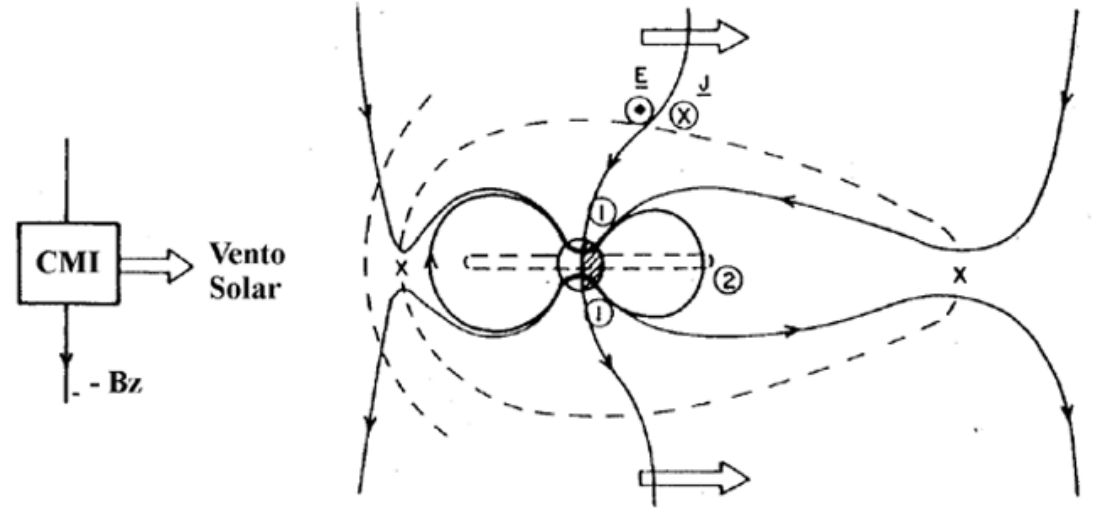
CMI : Campo Magnético Interplanetário
- Bz: Componente Sul do CMI
Ẹ: Campo Elétrico do Vento Solar
J: Corrente da Magnetopausa
E. $\underline{\text { J }}$ : Dínamo Magnetosférico
$\mathrm{x}$ : Região de Reconexão
(1): Dissipação Auroral
(2): Dissipação da Corrente de Anel

Figura 1 - Esquema do acoplamento Sol, Meio Interplanetário e Magnetosfera Terrestre. Demonstração do processo de reconexão e injeção de energia para dentro da Magnetosfera, 0 qual conduz a intensificação da Corrente de Anel (Gonzalez \& Tsurutani, 1992).

intensas (Dst <-200 nT) ocorridas no ano de 2003.

A seguir, analisamos dados de campo magnético e de plasma do meio interplanetário, que são medidos pelo satélite ACE (Advanced Composition Explorer) da NASA (National Aeronautics and Space Administration), localizado no ponto Lagrangeano L1, a fim de identificarmos as estruturas interplanetárias responsáveis por estas Tempestades Geomagnéticas Super Intensas (Dst < 200 nT). A bordo do satélite ACE temos o instrumento MAG ( Magnetic Field Experiment) que nos fornece observações do Campo Magnético Interplanetário $\left(B, B_{x}, B_{y}, B_{z}\right)$ e 0 instrumento SWEPAM (Solar Wind Electron Próton Alpha Monitor) que nos fornece observações de velocidade do Vento Solar, temperatura de prótons e densidade de prótons ( $V, T, N)$. Através dos dados do satélite ACE podemos classificar o tipo de estrutura e sua geoefetividade.

Os dados de raios cósmicos utilizados neste trabalho são do Telescópio Cintilador Multidirecional de Muons (Figura 2) que está instalado no município de São Martinho da Serra, RS, no Observatório Espacial do Sul OES/CRSPE/INPE-MCT (Latitude 29 , 26', 24”S, Longitude 53, 48', 38”0, altitude de $488 \mathrm{~m}$ acima do nível do mar).

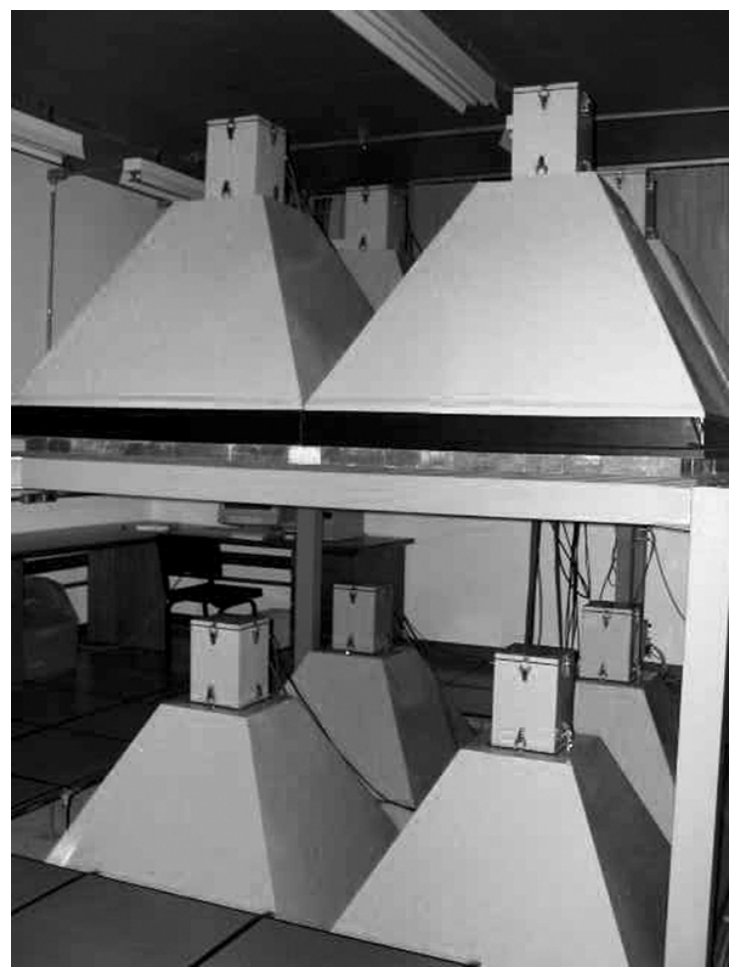

Figura 2 - Telescópio Cintilador Multidirecional de Muons instalado no OES/CRSPE/NNPE - MCT. 
Os dados do satélite ACE são disponibilizados pelo sistema CDA (Coordinate Data Analysis) do Programa ISTP (International Solar-Terrestrial Physics). Faremos uma descrição destas estruturas interplanetárias a fim de entender melhor a fenomenologia física envolvida.

\section{RESULTADOS E DISCUSSÕES}

Foram selecionados 3 eventos do meio interplanetário para este trabalho, os quais estão expostos na Tabela 1.

Tabela 1 - Eventos de Tempestades Geomagnéticas Intensas (Dst < -200 nT).

\begin{tabular}{|c|c|}
\hline Data & Dst (máximo) \\
\hline $30 / 10 / 2003$ & $-363 \mathrm{nT}$ \\
\hline $30 / 10 / 2003$ & $-401 \mathrm{nT}$ \\
\hline $20 / 11 / 2003$ & $-472 \mathrm{nT}$ \\
\hline
\end{tabular}

\section{Os eventos do dia $\mathbf{3 0}$ de Outubro de 2003}

A Figura 3 mostra um evento de choque interplanetário que ocorreu no dia 30 de Outubro de 2003. Temos de cima para baixo, a intensidade do campo magnético $\mathrm{B}$, a componente $\mathrm{z}$ do campo magnético $B_{z}$, a velocidade do vento solar $V$, a intensidade do índice Dst e as contagens relativas do canal vertical do Telescópio Cintilador Multidirecional de Muons V(\%).

Neste evento vemos no $3^{\circ}$ painel que há uma falha no equipamento SWEPAM (Solar Wind Electron Próton Alpha Monitor) que nos fornece dados de velocidade do vento solar (V), a qual pode ter sido causada pela incidência de partículas energéticas no satélite ACE, aceleradas por uma explosão solar relacionada a este evento.

A Figura 3 nos mostra a ocorrência de duas Tempestades Geomagnéticas Intensas cujo pico negativo do índice Dst da primeira chegou a $-363 \mathrm{nT}$, e da segunda a $-401 \mathrm{nT}$, tendo sido consideradas tempestades muito intensas.

A primeira linha vertical mostra 0 início da primeira tempestade no dia 30 de Outubro de 2003.

A estrutura interplanetária que causou esta tempestade foi uma nuvem magnética interplanetária, cuja principal característica é um campo magnético $B_{z}$ apresentando uma rotação de Sul para Norte (antes e depois da segunda linha). A componente do campo na direção Sul apresentou valor pico negativo de $-55 \mathrm{nT}$ e campo magnético pico total B de aproximadamente $60 \mathrm{nT}$. Apenas para referência, 0 valor típico de B no meio interplanetário nas proximidades da Terra é de $5 \mathrm{nT}$. A segunda linha marca a fase principal da tempestade e 0 início da fase de recuperação. A terceira linha mostra o final da primeira tempestade e início da se- gunda onde temos um pico negativo do Dst de $-401 \mathrm{nT}$. 0 campo magnético Bz na direção Sul foi de aproximadamente $-40 \mathrm{nT}$ e campo magnético $B$ de aproximadamente $40 \mathrm{nT}$. A quarta linha delimita a nuvem magnética e a fase de recuperação da segunda tempestade. A quinta linha indica o final da segunda tempestade. Há fortes indícios de que estas tempestades tenham sido causadas por duas fortes Ejeções Coronais de Massa observadas no Sol nos dias 28 e 29 de outubro, respectivamente.

No painel inferior podemos perceber um decréscimo nas contagens de muons observadas pelo TCM de São Martinho da Serra, de aproximadamente $15 \%$ na primeira tempestade e $6 \%$ na segunda. Uma característica interessante é que os dois decréscimos ocorrem na região entre 0 choque e a nuvem magnética, sugerindo que este seja causado pelo campo magnético turbulento desta região. Chama a atenção 0 fato de, mesmo se tratando de 2 eventos de proporções semelhantes, a resposta dos raios cósmicos observada pelo TCM de São Martinho da Serra ter sido consideravelmente diferente.

\section{Evento do dia 20 de Novembro de 2003}

A Figura 4 mostra um evento de choque interplanetário que ocorreu no dia 20 de Novembro de 2003. Temos de cima para baixo, a intensidade do campo magnético $B$, a componente $z$ do campo magnético $B_{z}$, a velocidade do vento solar $V$, a intensidade do índice Dste as contagens relativas do canal vertical do Telescópio Cintilador Multidirecional de Muons V(\%) do OES/CRSPE/INPE de São Martinho da Serra.

A Figura 4 nos mostra a ocorrência de uma Tempestade Geomagnética Intensa cujo pico negativo do índice Dst chegou a $-472 \mathrm{nT}$, considerada uma das maiores Tempestades Geomagnéticas da história do índice Dst.

A primeira linha mostra 0 início da tempestade no dia 20 de Novembro de 2003, a qual foi causada por uma nuvem magnética interplanetária proveniente de uma Ejeção Coronal de Massa. 0 campo magnético $B_{z}$ na direção Sul foi de aproximadamente -50 $\mathrm{nT}$ e campo magnético B de aproximadamente $60 \mathrm{nT}$. A segunda linha delimita a nuvem magnética e a fase de recuperação da tempestade. A terceira linha mostra o final da tempestade onde os parâmetros se estabilizam.

No painel inferior podemos perceber que 0 decréscimo na intensidade de muons é de aproximadamente 4\%. Do mesmo modo que no evento do dia 30 de Outubro de 2003 uma característica interessante é que o decréscimo ocorre na região entre o choque e a nuvem magnética, sugerindo que este seja causado pelo campo magnético turbulento desta região. A ordem de gran- 


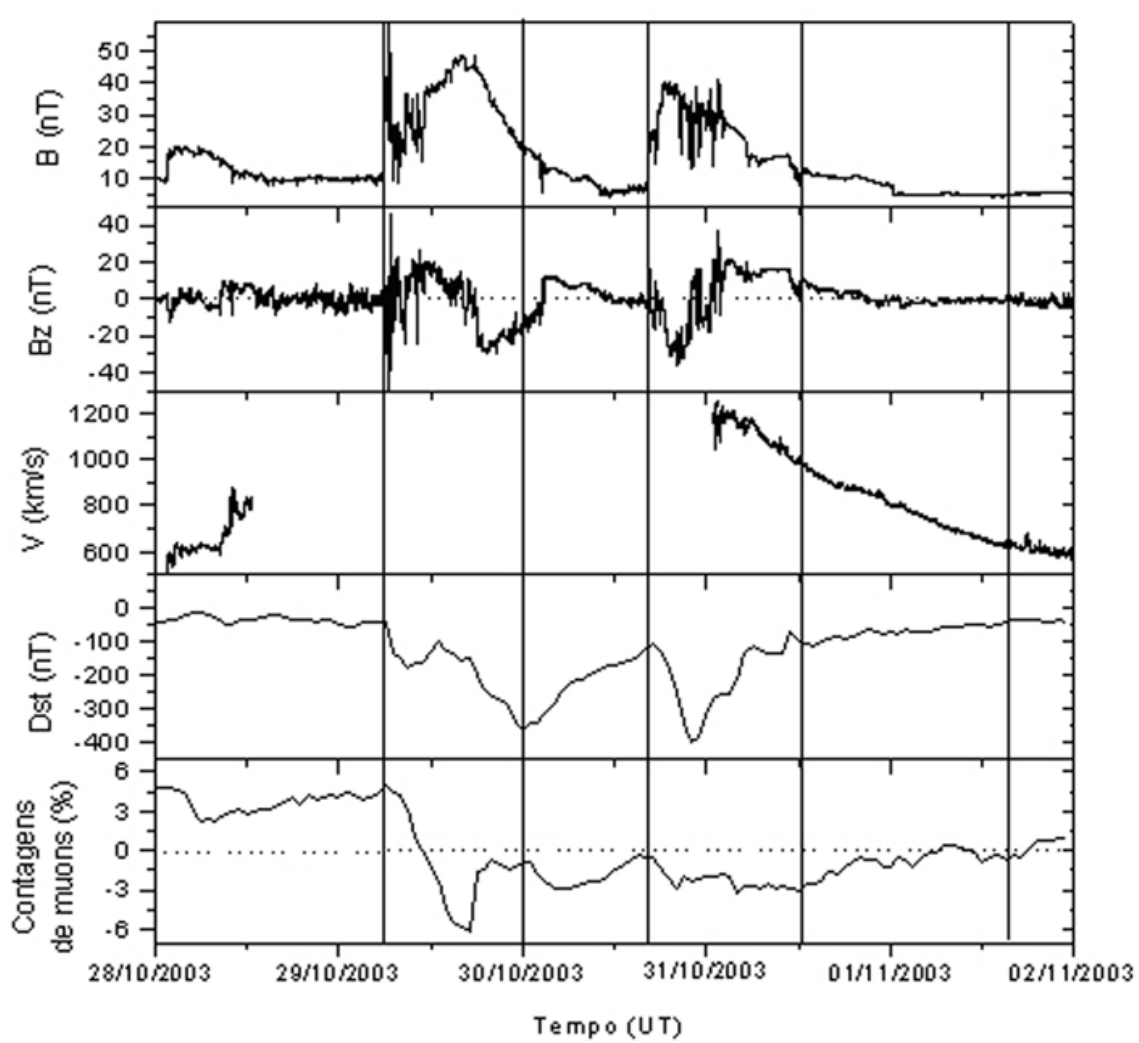

Figura 3 - Variaç̃ões temporais dos parâmetros físicos de uma estrutura do meio interplanetário, observada pelo satélite ACE em 30 de Outubro de 2003, variações do índice Dst e contagens relativas do Telescópio Cintilador Multidirecional de Muons do Observatório Espacial do Sul (OES/CRSPE/INPE - MCT).

deza dos parâmetros interplanetários e geomagnéticos envolvidos neste evento é semelhante aos 2 eventos observados no dia 30 de Outubro de 2003. A resposta dos raios cósmicos observados pelo TCM de São Martinho da Serra para este evento foi semelhante à observada para o segundo evento do dia 30 de Outubro de 2003.

\section{CONCLUSÕES}

Após fazermos uma análise das 3 Tempestades Geomagnéticas Super Intensas ocorridas no ano de 2003, analisando dados do índice Dst, do Meio Interplanetário e de raios cósmicos (muons) vimos que o canal vertical do TCM de São Martinho da Serra observou decréscimos em sua contagem durante os 3 eventos. Embora tenham sido eventos de proporções semelhantes, com valores de campo magnético interplanetário e resposta geomagnética de mesma ordem de grandeza, os raios cósmico não apresentaram respostas de igual intensidade para os 3 eventos, tendo sido a do primeiro evento do dia 30 de Outubro de 2003 consideravelmente maior que as 2 outras. Vimos que o decréscimo ocorre entre 0 choque e a nuvem magnética sugerindo que este tenha sido causado pelo campo magnético turbulento desta região. 0 monitoramento contínuo destes parâmetros faz-se necessário para que possamos entender melhor a resposta dos raios cósmicos de alta energia a fenômenos do meio interplanetário.

\section{AGRADECIMENTOS}

Os autores gostariam de agradecer ao pessoal de operação da missão ACE por fornecer dados do campo magnético interplanetário e vento solar, bem como ao pessoal de operação da Universidade de Kyoto, por fornecerem os dados interplanetários e de superfície utilizados neste trabalho. Os autores gostariam igualmente de agradecer ao Programa PIBIC/INPE - CNPq/MCT pela bolsa de Iniciação Científica, e ao CNPq pelo financiamento de parte deste trabalho através do Processo 472396/04-8.

\section{REFERÊNCIAS}

AKASOFU S \& CHAPMAN S. 1972. Solar Terrestrial Physics, Clarendon, Oxford, $901 \mathrm{p}$. 


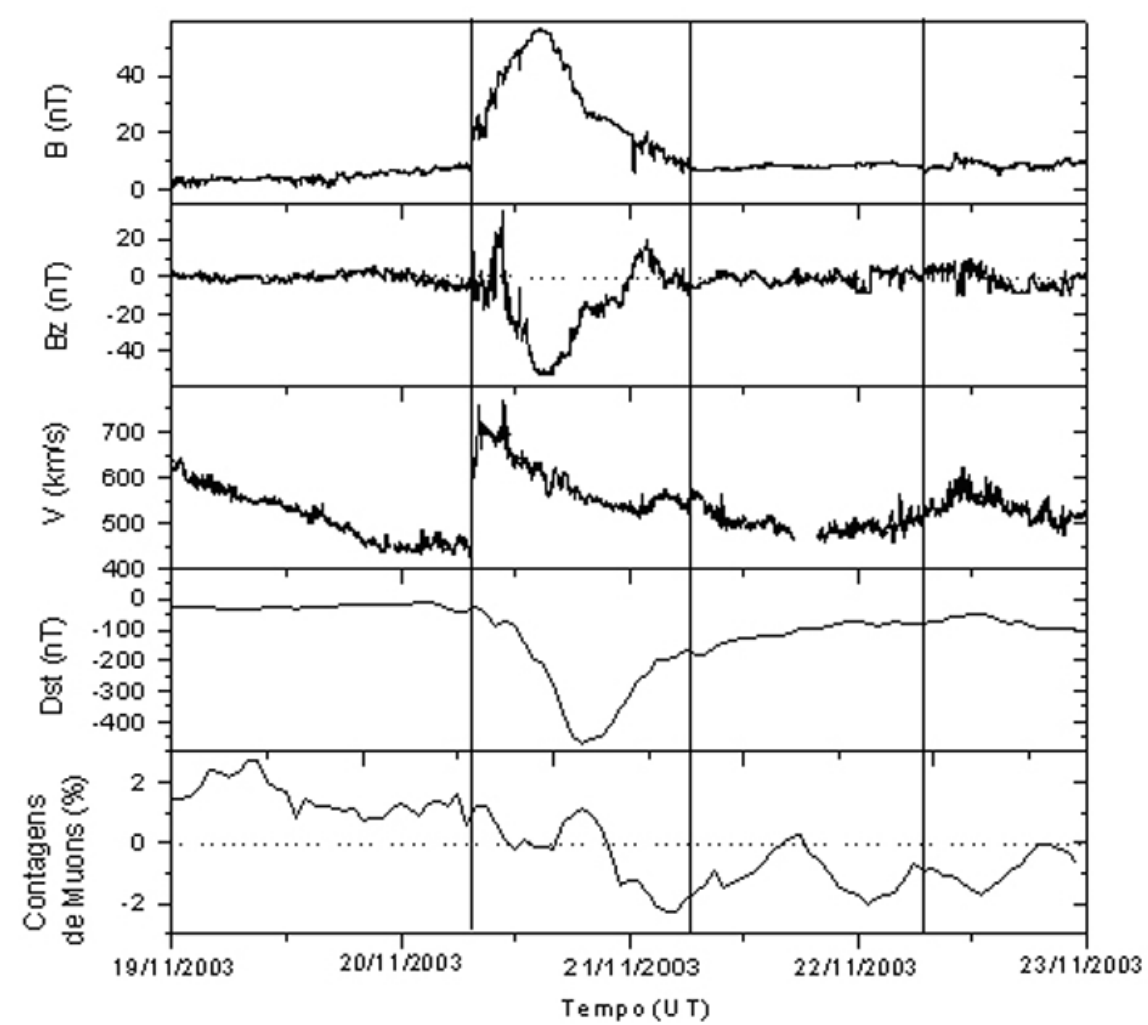

Figura 4 - Variações temporais dos parâmetros físicos de uma estrutura do Meio Interplanetário, observada pelo satélite ACE em 20 de Novembro de 2003, variações do índice Dst e contagens relativas do Telescópio Cintilador de Muons do Observatório Espacial do Sul (OES/CRSPE/INPE - MCT).

BURTON R, MCPHERRON R \& RUSSELL C. 1975. An empirical relationship between interplanetary conditions and Dst. J. Geophys. Res, 80: 4204.

DAL LAGO A, VIEIRA LEA, ECHER E, GONZALEZ WD, DE GONZALEZ ALC, GUARNIERI FL, BALMACEDA LA, SANTOS JC, DA SILVA MR, DE LUCAS A \& SCHUCH NJ. 2004. Great geomagnetic storms in the rise and maximum of solar cycle 23. Brazilian Journal of Physics, 34(4B): $1542-1546$

GONZALEZ WD \& TSURUTANI BT. 1987. Criteria of interplanetary causing intense magnetic storms (Dst < -100nT). Planet Space Sci, 35(9): 1101-1109.

GONZALEZ WD \& TSURUTANI BT. 1992. Terrestrial Response to Eruptive Solar Flares - Geomagnetic Storms, Eruptive Solar Flares. Proceedings of Colloquium \#133 of the International Astronomical Union, Publisher, Springer-Verlag, New York, p. 277.

GONZALEZ WD, JOSELYN JA, KAMIDE Y, KROEHL HW, ROSTOKER G, TSURUTANI BT \& VASYLIUNAS VM. 1994. What is a magnetic storm? J. Geophys. Res., 99(A4): 5771-5792.
JOKIPII JR. 2000. Cosmic Rays. In: SUESS ST \& TSURUTANI BT. (Eds.). From the Sun; Auroras, magnetic storms, solar flares, cosmic rays. Washington, DC: AGU, p. 123-131.

KAMIDE Y, YOKOYAMA N, GONZALEZ WD, TSURUTANI BT, DAGLIS I, BREKKE A \& MASUDA S. 1998. Two-step development of geomagnetic storms. J. Geophys. Res., 103(A4): 6917-6921.

LYONS L \& WILLIAMS DJ. 1980. A source for the geomagnetic storm main phase ring current, J. Geophys. Res., 85: 523-530.

NISHIDA A. 1978. Geomagnetic diagnosis of the magnetosphere. New York, Springer-Verlag, $256 \mathrm{p}$.

TSURUTANI BT \& GONZALEZ WD. 1997. The interplanetary cause of magnetic storms: a review. In: TSURUTANI BT, GONZALEZ WD, KAMIDE Y \& ARBALLO JA (Eds.). Magnetic storms, Washington, DC: AGU, 98: $77-89$.

TSURUTANI BT, GONZALEZ WD, TANG F \& LEE YT. 1992. Great magnetic storms. Geophys. Res. Lett., 19(1): 73-76. 


\section{NOTAS SOBRE OS AUTORES}

Jairo Francisco Savian é graduando em Física pela Universidade Federal de Santa Maria (UFSM), cursando o sexto semestre. Responsável em nível discente pelo Laboratório de Clima Espacial e Previsão de Tempestades Magnéticas do Centro Regional Sul do Instituto Nacional de Pesquisas Espaciais (CRSPE/INPE - MCT). Bolsista de Iniciação Científica do Conselho Nacional de Desenvolvimento Cientifico e Tecnológico (CNPq) desde Agosto de 2003, desenvolvendo a pesquisa intitulada: "Identificação das origens interplanetárias das Tempestades Geomagnéticas Intensas ocorridas no período de 2002 a 2003 ". Atualmente trabalha na coleta e tratamento dos dados do Telescópio Cintilador Multidirecional de Muons do Observatório Espacial do Sul (OES/CRSPE/INPE-MCT).

Marlos Rockenbach da Silva é graduado em Física pela Universidade Federal de Santa Maria (UFSM), mestre em Geofísica Espacial pelo Instituto Nacional de Pesquisas Espaciais (INPE), cujo título da dissertação é: "Variação da intensidade dos raios cósmicos em resposta a diferentes estruturas magnéticas do meio interplanetário".

Alisson Dal Lago é Licenciado em Física pela Universidade Federal de Santa Maria (1996), Mestre (1999) e Doutor (2003) em Geofísica Espacial pelo Instituto Nacional de Pesquisas Espaciais - INPE, Pesquisador Adjunto (desde 09/2004) da Divisão de Geofísica Espacial - DGE, Ciências Espaciais e Atmosféricas - CEA do INPE, onde trabalha na área de Clima Espacial, com previsão de ocorrência de distúrbios geomagnéticos utilizando observações espaciais e terrestres.

Kazuoki Munakata é Doutor em Física, Professor do Departamento de Física da Universidade de Shinshu, Japão, trabalha com pesquisa em raios cósmicos há cerca de 20 anos. Atualmente estuda a aplicação de observações de raios cósmicos para o Clima Espacial, sendo o coordenador, pela parte japonesa, da implantação da Rede de Detectores de Raios Cósmicos de Alta Energia, da qual o Telescópio Cintilador Multidirecional de Muons do Observatório Espacial do Sul - OES/CRSPE/INPE-MCT, de São Martinho da Serra, RS, Brasil, faz parte.

Walter Demetrio Gonzalez é graduado em Física pela Universidad Nacional de Ingenieria, UNI, Peru, Mestre em Geofísica Espacial pelo Instituto Nacional de Pesquisas Espaciais - INPE, Doutor em Física pela Universidade da Califórnia - Berkeley, Estados Unidos, Pesquisador Titular da Divisão de Geofísica Espacial - DGE, Ciências Espaciais e Atmosféricas - CEA do INPE, Chefe da Linha de Pesquisa em Magnetosfera e Heliosfera (MAGHEL) do INPE, Pesquisador 1A do CNPq. Estuda a origem interplanetária das Tempestades Geomagnéticas a mais de 20 anos, tendo publicado mais de 180 artigos sobre temas de Ciência Espacial.

Nelson Jorge Schuch é graduado em Física pela Universidade Federal de Santa Maria - UFSM, em 1972. Mestrado em Astrofísica pela Universidade Presbiteriana Mackenzie em 1975. Doutorado em Física, concentração - Astrofísica pela Universidade de Cambridge em 1979. Pós-Doutorado em Astrofísica pela Universidade de Cambridge em 1980. De 1980 a 1995, atuou como Pesquisador Titular/Vice-Diretor do Observatório Nacional. Em 1996 foi nomeado coordenador do Projeto Radioastronomia - RA, no âmbito da Parceria INPE - UFSM. É Chefe da Unidade Regional Sul de Pesquisas Espaciais e coordenador da ação 1275 e 6237 de implantação e funcionamento do Centro Regional Sul de Pesquisas Espaciais do PNAE, do Instituto Nacional de Pesquisas Espaciais. 